\title{
Correction to: Whole chromosome painting reveals independent origin of sex chromosomes in closely related forms of a fish species
}

\author{
Marcelo de Bello Cioffi ${ }^{1}$. Antonio Sánchez ${ }^{2} \cdot$ Juan Alberto Marchal ${ }^{2} \cdot$ Nadezda Kosyakova $^{3} \cdot$ Thomas Liehr $^{3}$. \\ Vladimir Trifonov ${ }^{4} \cdot$ Luiz Antonio Carlos Bertollo $^{1}$
}

Published online: 19 December 2017

(c) Springer International Publishing AG, part of Springer Nature 2017

\section{Correction to: Genetica (2011) 139:1065-1072 https://doi.org/10.1007/s10709-011-9610-0}

The Editorial Office of Genetica reports that a paragraph from the "Discussion" section of Cioffi et al. (2011; p. 1070, 4th paragraph of column 1) was transcribed (with only minor edits) from an introductory paragraph previously published in Chromosome Research by O'Meally et al. (2010, p. 788, 1st paragraph of the "Introduction" section). The corresponding author of the Genetica publication indicated that proper citation of the original source was inadvertently omitted. The paper published in Genetica by Cioffi et al. (2011) should have therefore included the citation of O'Meally et al. (2010), entitled "Non-homologous sex chromosomes of birds and snakes share repetitive sequences," and published in Chromosome Research (Chromosome Res, 2010; 18(7):787-800).

As a member of the Committee on Publication Ethics, Genetica is committed to ensure that original sources of information are properly acknowledged and cited in all of our publications.

Also in the original publication of the article, the family name of the corresponding author has been mistakenly processed as de Bello Cioffi. However, for publication and citation purposes, the family name should have been reported as Cioffi. This has now been updated in this correction article.

\section{Reference}

Cioffi MB, Sánchez A, Marchal JA, Kosyakova N, Liehr T, Trifonov V, Bertollo LAC (2011) Whole chromosome painting reveals independent origin of sex chromosomes in closely related forms of a fish species. Genetica 139:1065-1072

O’Meally D, Patel HR, Stiglec R, Sarre SD, Graves Marshall, Ezaz JA $\mathrm{T}$ (2010) Non-homologous sex chromosomes of birds and snakes share repetitive sequences. Chromosome Res 18:787-800

Communicated by Editors in Chief Juan L. Bouzat and Pierre Capy.

The online version of the original article can be found under https://doi.org/10.1007/s10709-011-9610-0.

Marcelo de Bello Cioffi

mbcioffi@ufscar.br

1 Departamento de Genética e Evolução, Universidade Federal de São Carlos, São Carlos, SP, Brazil

2 Departamento de Biología Experimental, Facultad de Ciencias Experimentales, Universidad de Jaén, Jaén, Spain

3 Institute of Human Genetics, Jena University Hospital, Kollegiengasse 10, 07743 Jena, Germany

4 Institute of Chemical Biology and Fundamental Medicine, SB RAS, Novosibirsk, Russia 